\title{
BMJ Open Two-field non-mydriatic fundus photography for diabetic retinopathy screening: a protocol for a systematic review and meta-analysis
}

\author{
Dongjing Yu, ${ }^{1,2,3}$ Xiaoyan Dou, ${ }^{4}$ Jiao Chen, ${ }^{2}$ Ying Lu, ${ }^{2}$ Baikang $Y e,{ }^{4}$ Xiaojun $\mathrm{Wu},{ }^{5}$ \\ Zijing Wu, ${ }^{2} \mathrm{Qi} \mathrm{Li},{ }^{1}$ Xiaohe Tian, ${ }^{2,6}$ Bo Zhou, ${ }^{2,7}$ Ying Deng, ${ }^{2,8}$ Wei $\mathrm{Li}^{2,9}$ Xinglin $\mathrm{Hu}^{2}{ }^{2}$ \\ Lisha Mou (i) , ${ }^{2}$ Zuhui $\mathrm{Pu}^{1}$
}

To cite: Yu D, Dou X, Chen J, et al. Two-field non-mydriatic fundus photography for diabetic retinopathy screening: a protocol for a systematic review and meta-analysis. BMJ Open 2021;11:e051761. doi:10.1136/ bmjopen-2021-051761

- Prepublication history and additional supplemental material for this paper are available online. To view these files, please visit the journal online (http://dx.doi.org/10.1136/ bmjopen-2021-051761)

DY and XD contributed equally.

Received 31 March 2021 Accepted 23 September 2021

Check for updates

(c) Author(s) (or their employer(s)) 2021. Re-use permitted under CC BY-NC. No commercial re-use. See rights and permissions. Published by BMJ.

For numbered affiliations see end of article.

Correspondence to Professor Lisha Mou; lishamou@gmail.com and Professor Zuhui Pu; pupeter190@163.com

\section{ABSTRACT}

Introduction Diabetic retinopathy (DR) is one of the most prevalent microvascular complications of diabetes mellitus. Guidelines for DR screening in different countries vary greatly, including fundus photography, slit-lamp biomicroscopy, indirect ophthalmoscopy, Optical Coherence Tomography (OCT), OCT-A and Fundus Fluorescein Angiography (FFA). Two-field non-mydriatic fundus photography (NMFP) is an effective screening method due to its low cost and less time-consuming process. However, it is controversial due to the sensitivity and specificity of two-field NMFP. This review intends to evaluate the performance of the two-field NMFP in diagnosing DR and helps clinicians determine the most optimal screening method.

Methods and analysis Two reviewers will independently search on the Medline, Embase, Cochrane databases, ProQuest, Opengrey, Chinese National Knowledge Infrastructure, Wanfang Data, VIP China Science and Technology Journal Database, Chinese BioMedical Literature Database, ISRCTN, ClinicalTrials.gov and the WHO ICTRP to identify relevant studies. There is no restriction posed on the language of the study. Included studies focus on the performance of two-field NMFP in detecting DR in diabetes patients. Analysis and evaluation of the studies will be examined by two reviewers independently using the Quality Assessment for Diagnostic Accuracy Studies-2 tool and later evaluated using the Population, Intervention, Comparison, Outcome, Study design criteria. A random-effect model will calculate the diagnostic indicators, including the sensitivity, specificity, positive likelihood ratio, negative likelihood ratio, diagnostic $\mathrm{OR}$, area under the curve and $95 \%$ Cls. We will also develop a summary receiver operating characteristic curve. We anticipate analysing subgroups according to the factors, which may lead to heterogeneity, including DR levels of patients, the reference standards, camera models, the interpretation criteria. The data will be analysed by STATA software. This study was registered with PROSPERO.

Ethics and dissemination This review will analyse the published data. Patients/the public were not involved in this research. The results of this study will be published in peer-reviewed journals.

PROSPERO registration number CRD42020203608.
Strengths and limitations of this study

- This study may provide the most appropriate screening methods and reliable, evidence-based medicine for diabetic retinopathy (DR).

- The heterogeneity has multiple sources, including different DR levels of patients imaged, the imaging procedure, the reference standards, camera models, ungradable images, the experience of the ophthalmologist, the interpretation criteria or a combination of these factors.

- All of the included studies used diagnostic casecontrol designs.

- Findings of the proposed systematic review and meta-analysis may be limited by publication bias, study heterogeneity and the methodological quality of existing research.

- We cannot guarantee that all relevant studies will be included in this meta-analysis.

\section{INTRODUCTION}

Diabetic retinopathy (DR) is one of the chronic complications of diabetes that causes cases of blindness among the working population. ${ }^{12}$ Blindness due to DR is one of the most feared complications and one of the most preventable. ${ }^{3}$ According to Global Vision Database, regarding the pooled rate of 14 global WHO study centres, about 3.2 million patients with DR were estimated to suffer from moderate or severe vision impairment by 2020.5

\section{Rationale}

Early DR develops relatively slowly; thus, the vision-threatening DR usually develops several years after the diagnosis of diabetes ${ }^{6}$ With a high prevalence of DR, early detection and treatment of DR are necessary for the diabetic population due to the poor prognosis of late DR. Early treatment can prevent $90 \%$ of severe vision loss. ${ }^{7}$ Blindness was also 


\begin{tabular}{|c|c|c|c|}
\hline Guidelines & Country & Screening methods & Ref \\
\hline 2020 AAO & USA & Slit-lamp biomicroscopy and indirect ophthalmoscopy & 12 \\
\hline $2017 \mathrm{AAO}$ & USA & Dilated comprehensive eye exam or retinal photography & 28 \\
\hline 2016 UK NSC & UK & Digital photography & 29 \\
\hline 2012 RCOphth & UK & $\begin{array}{l}\text { Digital photography (but selective mydriasis and numbers of fields are } \\
\text { controversial) }\end{array}$ & 30 \\
\hline 2018 Denmark & Denmark & Fundus photography or mydriatic with at least two fields & 31 \\
\hline 2017 SED/SEV & Spain & Non-mydriatic retinopathy or mydriatic retinopathy & 32 \\
\hline 2012 COS & Canada & Slit-lamp biomicroscopy and 7SF & 33 \\
\hline $2016 \mathrm{NZ}$ & New Zealand & Pupil dilation or colour digital retinal photography and slit-lamp biomicroscopy & 34 \\
\hline 2018 Poland & Poland & $\begin{array}{l}\text { Ophthalmoscope with mydriasis by an ophthalmologist or fundus camera by } \\
\text { trained personnel }\end{array}$ & 35 \\
\hline 2017 China & China & Two-field fundus photography (45-65 degrees) & 36 \\
\hline
\end{tabular}

DR, diabetic retinopathy; 7SF, 7-standard-field stereoscopic colour retinal imaging.

reduced significantly in Iceland, England and Wales through DR screening programs. ${ }^{8-11}$ However, $43 \%-65 \%$ of diabetic patients have not received a fundus examination when seeing a doctor. The fundus examinations promptly detect the vision-threatening symptoms of DR. Therefore, early prevention can be applied.

Multiple screening techniques can detect and classify DR. Guidelines for DR screening in different countries vary greatly. Although several other countries have screening guidelines, we choose several representative guidelines to show that the screening techniques vary greatly in different countries in table 1 . These stated guidelines show the divergent screening methods used in few countries. Not all the guidelines available are listed in the table. The latest published guideline by the American Academy of Ophthalmology in 2020 suggested using slit-lamp biomicroscopy and indirect ophthalmoscopy on DR detection. ${ }^{12}$ The UK National Screening Committee guideline established in 2016 sets up an adult screening programme for DR, while photographs of the back of the eyes are being used for diagnosis.

The 7-standard-field stereoscopic colour retinal imaging (7SF) is referred to as the gold standard in considerable research. Correspondingly, the Early Treatment Diabetic Retinopathy Study has used 7SF as the reference standard. ${ }^{13}$ Ophthalmologic slit-lamp biomicroscopy is also a commonly used detection method with high accuracy. Scanlon et al concluded that slit-lamp biomicroscopy by an ophthalmologist is a competitive method compared with $7 \mathrm{SF}$ for DR detection. ${ }^{14}$ However, these methods are too complex and tedious for primary care screening. Therefore, an effective and cost-efficient screening and detection strategy broadly accepted for DR is required.

Two-field non-mydriatic retinopathy is one of the costefficient screening and detection strategies on DR. ${ }^{15}$ Most accessible reviews evaluated the accuracy of several DR screening methods inclusively. For instance, Piyasena et al evaluated the DR test accuracy of two-field mydriatic retinopathy and two-field non-mydriatic fundus photography (NMFP). ${ }^{16}$ They examined the DR test accuracy by composing different combinations of the number of retinal fields, index test graders, pupil status and any possible confounding factors that might affect DR screening accuracy. However, only four studies of two-field NMFP were included in this analysis. Likewise, Bragge et al published a meta-analysis examining the effect of mydriasis and medical qualifications of photographers on the accuracy of DR screening. ${ }^{17}$ A systematic and comprehensive up-to-date analysis is required to address how effective the two-field NMFP is in diagnosing DR. Our meta-analysis aims to evaluate the non-mydriatic, two-field retinopathy exclusively regarding its accuracy and performance.

Pharmacological mydriasis usually results in blurred vision lasting several hours. Even though mydriasis significantly improves the technical failure rate of retinal photography, most patients refrain from doing eye examinations for DR screening. Because after mydriasis, they would not be able to work, drive or walk without assistance. Thus, undilated fundus photography is consequently favoured for screening.

Single-field NMFP is considered the simplest screening method in the primary care setting, but it did not meet the British Diabetic Association (BDA) standard according to our previous meta-analysis study. ${ }^{18}$ According to BDA, the screening method for DR needs to achieve at least $80 \%$ sensitivity and $95 \%$ specificity. ${ }^{19}$ Admittedly, sufficient studies proved that single-field NMFP does not have the required sensitivity and specificity. Two-field NMFP is being studied and evaluated as to whether it is an effective screening method in certain research. However, it is controversial as far as the sensitivity and specificity of two-field NMFP are concerned. Saari et al reported that the sensitivity $(88.9 \%)$ and specificity $(100 \%)$ of twofield NMFP met the technical requirements of BDA. ${ }^{20}$ But Boucher et al and Perrier et al reported the sensitivity $(97.7 \%, 95.7 \%$, respectively) and specificity $(84.0 \%$, $75.8 \%$, respectively). ${ }^{21} 22$ The specificity of two-field 
NMFP in these studies did not meet the BDA standard of more than $95 \%$ specificity. Furthermore, the different performances of two-field NMFP for DR detection evoked much controversy. Previous systematic reviews and metaanalyses include only four studies with limited samples. Thus, there is an urgent need to determine whether it is an effective screening strategy through an up-to-date systematic review and meta-analysis.

\section{Objective}

This study aims to evaluate the effectiveness of two-field NMFP in DR detection by performing a meta-analysis. The main objective of the systematic review purposes is to facilitate physicians in determining the most appropriate screening tool for DR.

\section{METHODS AND ANALYSIS}

The systematic review and meta-analysis will be prepared according to a prespecified protocol registered with PROSPERO (without peer review) for the Preferred reporting items for systematic review and meta-analysis protocols 2015 statement. ${ }^{23}$

\section{Inclusion criteria}

Two reviewers will independently examine all the acquired studies and evaluate them using the information provided in the title and abstract. The studies will be eligible for this study only if they met the following PICOS criteria (table 2): (1) Population (P), type 1 or/and type 2 diabetes patients known or suspected to have any level of DR; (2) Intervention (I), two-field NMFP focusing on two centres as a screening method for DR, photographs are graded by ophthalmologists and provided with the original data; (3) Comparison (C), standard diagnostic methods (eg, 7SF, slit-lamp biomicroscopy, FFA); (4) Outcome $(\mathrm{O})$, diagnostic accuracy of Two-Field NMFP for $\mathrm{DR}$, using 7SF or slit-lamp biomicroscopy as the reference standard; (5) Study design (S), prospective case-control studies. The study setting includes all kinds of settings, for example, major hospitals, primary care settings, etc.

\begin{tabular}{|c|c|}
\hline PICOS & Description \\
\hline Population (P) & Patients known or suspected to have DR \\
\hline Intervention (I) & $\begin{array}{l}\text { Two-field NMFP as a screening method } \\
\text { for diabetic retinopathy }\end{array}$ \\
\hline Comparison (C) & $\begin{array}{l}\text { Standard diagnostic methods (7SF, slit- } \\
\text { lamp biomicroscopy, FFA) }\end{array}$ \\
\hline Outcome (O) & $\begin{array}{l}\text { Diagnostic accuracy of two-field NMFP for } \\
\text { DR, using } 7 \text { SF or slit-lamp biomicroscopy } \\
\text { as the reference standard }\end{array}$ \\
\hline
\end{tabular}

Study design (S) Prospective case-control studies

DR, diabetic retinopathy; NMFP, non-mydriatic fundus photography; 7SF, 7-standard-field stereoscopic colour retinal imaging.

\section{Literature search}

Two reviewers (DY and LM) independently screened all the acquired studies based on the title and abstract information. After that, the full text of the selected articles will be evaluated for eligibility. The consensus of the two reviewers will resolve any discrepancy.

We will search the Medline Ovid, Embase Ovid, Cochrane databases, ProQuest, Opengrey, Chinese National Knowledge Infrastructure (CNKI), Wanfang Data, VIP China Science and Technology Journal Database (VIP), Chinese BioMedical Literature Database (CBM), ISRCTN, ClinicalTrials.gov and the WHO ICTRP to identify studies updated to the time we search. There will be no restriction based on language. In addition, we will use a combination of medical subject headings and text terminologies to define the study population (people with diabetes), the pathological process of interest (DR) and the specific screening techniques used (fundus photography or digital retinal imaging system). The duplicates will be removed, and each article is screened based on its title and abstract. Subsequently, we will assess the full text of studies and include only relevant studies in our meta-analysis. The search strategy was described in online supplemental file S1. We will also examine the reference lists in articles to obtain additional studies not obtained by the electronic search.

\section{Quality assessment}

Two reviewers (DY and LM) will independently assess the quality of the selected studies using the Quality Assessment for Diagnostic Accuracy Studies (QUADAS-2). ${ }^{24}$ QUADAS-2 intends to assess the quality of primary diagnostic accuracy studies by evaluating the risk of bias and applicability in patient selection, the index test, and the reference standard will be used for study flow and timing. We will then use these assessments to evaluate the risk of bias of all the included studies: (1) low, if there are no risks of bias in four key domains; (2) unclear, if there is not enough information provided for the assessment of the risk of bias; (3) high, if the risk of bias is high for one or more key domains. Disagreements will be discussed with a third reviewer and will be further resolved by consensus.

\section{Data extraction}

Two reviewers (DY and LM) will extract the characteristics independently from the included studies, including (1) true positives, (2) false positives, (3) true negatives, (4) false negatives, (5) sampling method, (6) sample size, (7) country, (8) age, (9) gender, (10) diabetes type, (11) duration of disease, (12) camera model, (13) reference test into an Excel sheet. Disagreements and discrepancies will be discussed with a third reviewer and resolved by consensus. The search and data extraction will be done in 6 months after this protocol is accepted. The data will be extracted into an Excel sheet.

\section{Data analysis}

The data synthesis and analysis evaluate the diagnostic performance and accuracy of the two-field NMFP in 
detecting any DR. We will analyse data for eyes, but ungradable images will be excluded. The statistical analysis will be performed by STATA V.16.0. We will calculate the following outcome: sensitivity, specificity, positive likelihood ratio, negative likelihood ratio and the diagnostic OR with $95 \%$ CIs. If high heterogeneity is induced, we will likely use the random-effects model as opposed to a fixedeffect model to assess the statistical heterogeneity among all included studies. Consecutively, we will consider the $\mathrm{I}^{2}$ statistic to evaluate the heterogeneity in all the studies. In general, a higher percentage of $\mathrm{I}^{2}$ indicates increasing heterogeneity. Moreover, $\mathrm{I}^{2}>50 \%(\mathrm{p}=0.05)$ will be considered statistical heterogeneity. ${ }^{25}$ Besides, we will perform the receiver-operating characteristic (ROC) curves and use the sensitivity, specificity and area under the curve to facilitate statistical analysis. ${ }^{26}$ If there are more than ten studies are included, we will take the summary operating point on the summary ROC (SROC) curve as the estimate of the test performance among all studies in our meta-analysis. Thus, we will extrapolate the $Q$ values, when specificity equals sensitivity on the SROC curve, to further evaluate heterogeneity. Funnel plots will be used to visualise publication bias in meta-analysis. We will plot on a logarithm scale using SE and the effect measure. Moreover, the heterogeneity in all studies will be considered in the analysis. We anticipate analysing subgroups according to the factors potentially leading to heterogeneity, including different DR levels of patients imaged, the imaging procedure, the reference standards (eg, 7SF, slit-lamp biomicroscopy, FFA), camera models, ungradable images, the experience of the ophthalmologist, the interpretation criteria. If a high degree of heterogeneity occurs, we will present the findings narratively.

\section{Patient and public involvement}

Patients were not involved in the design of this study.

\section{Ethics and dissemination}

Since we will not collect primary data of individual patients, there is no need for ethical approval. The final results of this analysis will be published in a peer-reviewed journal. It will provide evidence of accuracy on two-field NMFP to the ophthalmologists or any healthcare workers in the primary clinic setting.

\section{Amendments}

The protocol for this study will be amended if new guidelines for DR screening are released during preparation.

\section{DISCUSSION}

It remains controversial whether two-field NMFP is efficient enough to be a DR screening method according to BDA standard. ${ }^{22}{ }^{27}$ DR and its levels were crucial for clinical studies to make therapeutic methods and avoid further progression. Therefore, this meta-analysis aims to collate comprehensive, up-to-date evidence concerning the diagnostic test accuracy of two-field NMFP for DR detection, to answer whether two-field NMFP is efficient enough to be a DR screening method, according to BDA standards.

The results may have important practical implications for clinicians, patients with DR and those working on DR research. Our findings will also be expected to provide evidence for clinical decision support for DR screening. It can also guide the DR screening method for healthcare professionals. To this end, the results of this systematic review and meta-analysis will be published in a peer-reviewed journal, potentially benefitting healthcare professionals, patients and guideline makers.

\section{Author affiliations}

${ }^{1}$ Imaging Department, Shenzhen Institute of Translational Medicine, The First Affiliated Hospital of Shenzhen University, Shenzhen Second People's Hospital, Shenzhen, China

${ }^{2}$ Shenzhen Xenotransplantation Medical Engineering Research and Development Center, Shenzhen Institute of Translational Medicine, The First Affiliated Hospital of Shenzhen University, Shenzhen Second People's Hospital, Shenzhen, People's Republic of China

${ }^{3}$ Department of Life Sciences, University of Toronto, Toronto, Ontario, Canada ${ }^{4}$ Department of Ophthalmology, Shenzhen Institute of Translational Medicine, The First Affiliated Hospital of Shenzhen University, Shenzhen Second People's Hospital, Shenzhen, People's Republic of China

${ }^{5}$ Department of Ophthalmology, Shenzhen Nanshan People's Hospital, 6th Affiliated Hospital of Shenzhen University Health Science Center, Shenzhen, China

${ }^{6}$ Rausser College of Natural Resources, University of California Berkeley, Berkeley, California, USA

${ }^{7}$ College of Engineering, Boston University, Boston, Massachusetts, USA

${ }^{8}$ Faculty of Science, University of Waterloo, Waterloo, Ontario, Canada

${ }^{9}$ College of Science, Northeastern University, Boston, Massachusetts, USA

Contributors ZP conceived the idea, planned, conceived and designed the protocol. LM designed and piloted the search strategy. DY, XD, JC, YL, ZW, XH, XT, $B Z, Y D, W L, B Y, X W, Q L$ critically reviewed and provided feedback. LM and DY wrote the manuscript. $X D, B Y, X W$ and $L M$ revised the manuscript. $Z P$ and $L M$ are the guarantors of the review.

Funding This work was supported by Shenzhen High-level Hospital Construction Fund (2019), grant number not applicable, Clinical Research Project of Shenzhen Second People's Hospital (grant number: 20193357009).

Competing interests None declared.

Patient consent for publication Not applicable.

Provenance and peer review Not commissioned; externally peer reviewed.

Supplemental material This content has been supplied by the author(s). It has not been vetted by BMJ Publishing Group Limited (BMJ) and may not have been peer-reviewed. Any opinions or recommendations discussed are solely those of the author(s) and are not endorsed by BMJ. BMJ disclaims all liability and responsibility arising from any reliance placed on the content. Where the content includes any translated material, BMJ does not warrant the accuracy and reliability of the translations (including but not limited to local regulations, clinical guidelines, terminology, drug names and drug dosages), and is not responsible for any error and/or omissions arising from translation and adaptation or otherwise.

Open access This is an open access article distributed in accordance with the Creative Commons Attribution Non Commercial (CC BY-NC 4.0) license, which permits others to distribute, remix, adapt, build upon this work non-commercially, and license their derivative works on different terms, provided the original work is properly cited, appropriate credit is given, any changes made indicated, and the use is non-commercial. See: http://creativecommons.org/licenses/by-nc/4.0/.

ORCID iD

Lisha Mou http://orcid.org/0000-0001-6232-8341 


\section{REFERENCES}

1 Wykoff CC, Khurana RN, Nguyen QD, et al. Risk of blindness among patients with diabetes and newly diagnosed diabetic retinopathy. Diabetes Care 2021;44:748-56.

2 Yau JWY, Rogers SL, Kawasaki R, et al. Global prevalence and major risk factors of diabetic retinopathy. Diabetes Care 2012;35:556-64.

3 Bourne RRA, Stevens GA, White RA, et al. Causes of vision loss worldwide, 1990-2010: a systematic analysis. Lancet Glob Health 2013;1:e339-49.

4 World Health Organization. Diabetes: key facts, 2018. Available: https://www.who.int/en/news-room/fact-sheets/detail/diabetes

5 Flaxman SR, Bourne RRA, Resnikoff S, et al. Global causes of blindness and distance vision impairment 1990-2020: a systematic review and meta-analysis. Lancet Glob Health 2017;5:e1221-34.

6 Diabetes Control and Complications Trial Research Group, Nathan DM, Genuth S, et al. The effect of intensive treatment of diabetes on the development and progression of long-term complications in insulin-dependent diabetes mellitus. N Engl J Med 1993;329:977-86.

7 Vashist P, Singh S, Gupta N, et al. Role of early screening for diabetic retinopathy in patients with diabetes mellitus: an overview. Indian $J$ Community Med 2011;36:247-52.

8 Scanlon $\mathrm{PH}$. The English national screening programme for diabetic retinopathy 2003-2016. Acta Diabetol 2017;54:515-25.

9 Olafsdóttir E, Stefánsson E. Biennial eye screening in patients with diabetes without retinopathy: 10-year experience. $\mathrm{Br} J$ Ophthalmol 2007;91:1599-601.

10 Zoega GM, Gunnarsdóttir T, Björnsdóttir S, et al. Screening compliance and visual outcome in diabetes. Acta Ophthalmol Scand 2005;83:687-90.

11 Liew G, Michaelides M, Bunce C. A comparison of the causes of blindness certifications in England and Wales in working age adults (16-64 years), 1999-2000 with 2009-2010. BMJ Open 2014;4:e004015.

12 Flaxel CJ, Adelman RA, Bailey ST, et al. Diabetic retinopathy preferred practice Pattern $\circledast$. Ophthalmology 2020;127:P66-145.

13 Grading diabetic retinopathy from stereoscopic color fundus photographs--an extension of the modified Airlie House classification. ETDRS report number 10. Early Treatment Diabetic Retinopathy Study Research Group. Ophthalmology 1991;98:786-806.

14 Scanlon $\mathrm{PH}$, Malhotra R, Greenwood RH, et al. Comparison of two reference standards in validating two field mydriatic digital photography as a method of screening for diabetic retinopathy. $\mathrm{Br} J$ Ophthalmol 2003;87:1258-63.

15 Lopez-Bastida J, Cabrera-Lopez F, Serrano-Aguilar P. Sensitivity and specificity of digital retinal imaging for screening diabetic retinopathy. Diabet Med 2007;24:403-7.

16 Piyasena MMPN, Murthy GVS, Yip JLY, et al. Systematic review and meta-analysis of diagnostic accuracy of detection of any level of diabetic retinopathy using digital retinal imaging. Syst Rev 2018;7:182.

17 Bragge P, Gruen RL, Chau M, et al. Screening for presence or absence of diabetic retinopathy: a meta-analysis. Arch Ophthalmol 2011;129:435-44.

$18 \mathrm{Hu}$ J, Chen R, Lu Y, et al. Single-Field Non-Mydriatic fundus photography for diabetic retinopathy screening: a systematic review and meta-analysis. Ophthalmic Res 2019;62:61-7.

19 British Diabetic Association. Retinal photography screening for diabetic eye disease, 1997.
20 Saari JM, Summanen P, Kivelä T, et al. Sensitivity and specificity of digital retinal images in grading diabetic retinopathy. Acta Ophthalmol Scand 2004;82:126-30.

21 Boucher MC, Gresset JA, Angioi K, et al. Effectiveness and safety of screening for diabetic retinopathy with two nonmydriatic digital images compared with the seven standard stereoscopic photographic fields. Can J Ophthalmol 2003;38:557-68.

22 Perrier M, Boucher MC, Angioi K, et al. Comparison of two, three and four 45 degrees image fields obtained with the Topcon CRW6 nonmydriatic camera for screening for diabetic retinopathy. Can $\mathrm{J}$ Ophthalmol 2003;38:569-74.

23 Moher D, Shamseer L, Clarke M, et al. Preferred reporting items for systematic review and meta-analysis protocols (PRISMA-P) 2015 statement. Syst Rev 2015;4:1.

24 Whiting PF, Rutjes AWS, Westwood ME, et al. QUADAS-2: a revised tool for the quality assessment of diagnostic accuracy studies. Ann Intern Med 2011;155:529-36.

25 Higgins JPT, Thompson SG, Deeks JJ, et al. Measuring inconsistency in meta-analyses. BMJ 2003;327:557-60.

26 Hajian-Tilaki K. Receiver operating characteristic (ROC) curve analysis for medical diagnostic test evaluation. Caspian $\mathrm{J}$ Intern Med 2013;4:627-35.

27 Pugh JA, Jacobson JM, Van Heuven WA, et al. Screening for diabetic retinopathy. The wide-angle retinal camera. Diabetes Care 1993;16:889-95.

28 American Academy of Ophthalmology. Diabetic retinopathy preferred practice Pattern $\AA$, 2017. Available: https://www.aao.org/preferredpractice-pattern/diabetic-retinopathy-ppp [Accessed 21 Feb 2021]

29 UK National Screening Committee. Adult screening programme diabetic retinopathy, 2016. Available: https://view-health-screeningrecommendations.service.gov.uk/diabetic-retinopathy/ [Accessed 2021.10.12].

30 The Royal College of Ophthalmologists. Diabetic retinopathy guidelines, 2012. Available: http://www.icoph.org/dynamic/ attachments/taskforce_documents/2012-sci-267_diabetic retinopathy guidelines december_2012.pdf [Accessed 2021.10.12].

31 Grauslund J, Andersen N, Andresen J, et al. Evidence-based Danish guidelines for screening of diabetic retinopathy. Acta Ophthalmol 2018;96:763-9.

32 Corcóstegui B, Durán S, González-Albarrán MO, et al. Update on diagnosis and treatment of diabetic retinopathy: a consensus guideline of the Working group of ocular health (Spanish Society of diabetes and Spanish vitreous and retina Society). J Ophthalmol 2017;2017:1-10.

33 Canadian Ophthalmological Society Diabetic Retinopathy Clinical Practice Guideline Expert Committee, Hooper P, Boucher MC, et al. Canadian Ophthalmological Society Evidence-based Clinical Practice Guidelines for the Management of Diabetic Retinopathy executive summary. Can J Ophthalmol 2012;47:91-101.

34 Ministry of Health. Diabetic retinal screening, grading, monitoring and referral guidance, 2016. Available: https://www.health.govt.nz/ system/files/documents/publications/diabetic-retinal-screeninggrading-monitoring-referral-guidance-mar16.pdf [Accessed 2021.10.12].

35 Diabetes Poland (Polish Diabetes Association). 2018 guidelines on the management of diabetes patients: clinical Diabetology, 2018.

36 Chinese Ophthalmological Society. Guidelines for image collection and reading of screening for diabetic retinopathy in China (2017). Chin J Ophthalmol 2017;53:890-6. 